\title{
MEROMORPHIC FUNCTIONS THAT SHARE THREE SETS
}

\author{
HoNG-Xun YI
}

\begin{abstract}
In this paper, we deal with the problem of uniqueness of meromorphic functions that share three sets and obtain some unicity theorems which improve some theorems given by F. Gross and C. F. Osgood, K. Tohge, G. Brosch, G. Jank and N. Terglane, H. X. Yi and other authors.
\end{abstract}

\section{Introduction and main results}

By a meromorphic function we shall always mean a meromorphic function in the complex plane $\boldsymbol{C}$. It is assumed that the reader is familiar with the notations of the Nevanlinna theory that can be found, for instance, in [1]. Let $h$ be a nonconstant meromorphic function and let $S$ be a subset of distinct elements in $\hat{C}=\boldsymbol{C} \cup\{\infty\}$. Define

$$
E_{h}(S)=\bigcup_{a \in S}\{z \mid h(z)-a=0\},
$$

where each zero of $h(z)-a$ (or $1 / h(z)$ if $a=\infty$ ) with multiplicity $m$ is repeated $m$ times in $E_{h}(S)$ (see [2]). The notation $\bar{E}_{h}(S)$ expresses the set which contains the same points as $E_{h}(S)$ but without counting multiplicities.

Let $f$ and $g$ be two nonconstant meromorphic functions and $S$ be a subset of distinct elements in $\hat{\boldsymbol{C}}$. If $E_{f}(S)=E_{g}(S)$, we say $f$ and $g$ share the set $S$ CM (counting multiplicity). If $\vec{E}_{f}(S)=\vec{E}_{\mathrm{g}}(S)$, we say $f$ and $g$ share the set $S$ IM (ignoring multiplicity). As a special case, let $S=\{a\}$, where $a \in \hat{C}$. If $E_{f}(\{a\})=$ $E_{g}(\{a\})$, we say $f$ and $g$ share the value $a \mathrm{CM}$. If $\bar{E}_{f}(\{a\})=\bar{E}_{g}(\{a\})$, we say $f$ and $g$ share the value $a$ IM (see [3]).

In 1982, F. Gross and C. F. Osgood proved the following theorem.

THeOREM A [4]. Let $S_{1}=\{-1,1\}, S_{2}=\{0\}$. If $f$ and $g$ are nonconstant entire functions of finite order such that $f$ and $g$ share the sets $S_{1}$ and $S_{2} C M$, then $f \equiv \pm g$ or $f \cdot g \equiv \pm 1$.

Project supported by the National Natural Science Foundation of China.

1991 Mathematics Subject Classification. Primary 30D35, Secondary 30D30, 30D20.

Key words and phrases. Nevanlinna theory, meromorphic function, shared set, uniqueness theorem.

Received April 16, 1996; revised August 20, 1996. 
In 1987, the present author [5] proved that in the preceding theorem the order restriction of $f$ and $g$ can be removed and obtained the following result.

Theorem B [5]. Let $S_{1}=\{-1,1\}, S_{2}=\{0\}, S_{3}=\{\infty\}$. If $f$ and $g$ are nonconstant meromorphic functions such that $f$ and $g$ share the sets $S_{j}(j=1,2,3)$ $C M$, then $f \equiv \pm g$ or $f \cdot g \equiv \pm 1$.

In 1989, G. Brosch [6] also independently proved Theorem B.

Unless stated otherwise, in the following theorems, $f$ and $g$ are two nonconstant meromorphic functions, $S_{1}=\left\{1, \omega, \ldots, \omega^{n-1}\right\}, S_{2}=\{0\}, S_{3}=\{\infty\}$, where $n$ is a positive integer, $\omega$ denotes the constant $\cos (2 \pi / n)+i \sin (2 \pi / n)$.

In 1988, the present author [7] and independently K. Tohge [8] proved the following theorem which is an extension of the above results. then

THEOREM C. Suppose that $f$ and $g$ share the sets $S_{j}(j=1,2,3)$ CM. If $n \geq 2$,

$$
f \equiv \operatorname{tg}
$$

where $t^{n}=1$ or

$$
f \cdot g \equiv s
$$

where 0 and $\infty$ are lacunary values of $f$ and $g$, and $s^{n}=1$.

In 1990, the present author [9] gives a short proof of Theorem C.

In 1991, G. Jank and N. Terglane [10] proved the following theorem, which is an improvement of Theorem $\mathrm{C}$.

THEOREM D. Suppose that $f$ and $g$ share the sets $S_{1}$ and $S_{2} C M$ and $S_{3}$ IM. If $n \geq 2$, then $f$ and $g$ satisfy (1.1) or (1.2).

In this paper, we prove the following results which are improvements and supplements of the above theorems.

THEOREM 1. Suppose that $f$ and $g$ share the sets $S_{1}$ and $S_{3} C M$ and $S_{2}$ IM. If $n \geq 2$, then $f$ and $g$ satisfy (1.1) or (1.2).

THEOREM 2. Suppose that $f$ and $g$ share the sets $S_{1} C M$ and $S_{2}$ and $S_{3}$ IM. If $n \geq 3$, then $f$ and $g$ satisfy (1.1) or (1.2).

THEOREM 3. Suppose that $f$ and $g$ share the sets $S_{2} C M$ and $S_{1}$ and $S_{3} I M$. If $n \geq 6$, then $f$ and $g$ satisfy (1.1) or (1.2).

THEOREM 4. Suppose that $f$ and $g$ share the sets $S_{3} C M$ and $S_{1}$ and $S_{2}$ IM. If $n \geq 6$, then $f$ and $g$ satisfy (1.1) or (1.2). 
From Theorem 4 we immediately obtain the following:

Corollary. Suppose that $f$ and $g$ share the sets $S_{2}$ and $S_{3} C M$ and $S_{1}$ IM. If $n \geq 6$, then $f$ and $g$ satisfy (1.1) or (1.2).

THEOREM 5. Suppose that $f$ and $g$ share the sets $S_{1}, S_{2}$ and $S_{3}$ IM. If $n \geq 7$, then $f$ and $g$ satisfy (1.1) or (1.2).

THEOREM 6. Suppose that $f$ and $g$ are two nonconstant entire functions such that $f$ and $g$ share the sets $S_{1}$ and $S_{2}$ IM. If $n \geq 4$, then $f$ and $g$ satisfy (1.1) or (1.2).

\section{Some lemmas}

LEMMA 1 [3]. Let $F$ and $G$ be two nonconstant meromorphic functions such that $F$ and $G$ share $1,0, \infty I M$, then

$$
T(r, F)=O(T(r, G)), \quad T(r, G)=O(T(r, F)),
$$

possibly outside a set of finite Lebesgue measure.

Remark. From Lemma 1, we can see that if $F$ and $G$ share $1,0, \infty$ IM, then $S(r ; G)=S(r, F)$. For simplicity, we denote them by $S(r)$ in the following discussion.

Lemma 2 [11]. Let $h$ be a nonconstant meromorphic function, then

$$
N_{o}\left(r, \frac{1}{h^{\prime}}\right) \leq \bar{N}\left(r, \frac{1}{h}\right)+\bar{N}(r, h)+S(r, h),
$$

where $N_{0}\left(r, 1 / h^{\prime}\right)$ denotes the counting function corresponding to the zeros of $h^{\prime}$ that are not zeros of $h$.

Remark. It is easy to give the proof of Lemma 2. In fact, let

$$
H=\frac{h^{\prime}}{h} \text {. }
$$

$$
N_{0}\left(r, \frac{1}{h^{\prime}}\right) \leq N\left(r, \frac{1}{H}\right) \leq T(r, H)+O(1) \leq \bar{N}\left(r, \frac{1}{h}\right)+\bar{N}(r, h)+S(r, h),
$$

which proves Lemma 2.

Next, we introduce the following notations.

Let $F$ and $G$ be two nonconstant meromorphic functions such that $F$ and $G$ share 1 IM. Let $z_{0}$ be a 1-point of $F$ of order $p$, a 1-point of $G$ of order $q$. We denote by $\bar{N}_{S}(r, 1 /(F-1))$ the counting function of those 1-points of $F$ where 
$p<q ; \bar{N}_{E}(r, 1 /(F-1))$ the counting function of those 1-points of $F$ where $p=q$; $\bar{N}_{L}(r, 1 /(F-1))$ the counting function of those 1-points of $F$ where $p>q$; each point in these counting functions is counted only once. In the same way, we can define $\bar{N}_{S}(r, 1 /(G-1)), \bar{N}_{E}(r, 1 /(G-1))$ and $\bar{N}_{L}(r, 1 /(G-1))$ (see [12] or [13]). Particularly, if $F$ and $G$ share $1 \mathrm{CM}$, then

$$
\bar{N}_{S}\left(r, \frac{1}{F-1}\right)=\bar{N}_{L}\left(r, \frac{1}{F-1}\right)=\bar{N}_{S}\left(r, \frac{1}{G-1}\right)=\bar{N}_{L}\left(r, \frac{1}{G-1}\right)=0
$$

With these notations, it is easy to see that

$$
\begin{aligned}
& \bar{N}_{S}\left(r, \frac{1}{F-1}\right)=\bar{N}_{L}\left(r, \frac{1}{G-1}\right) \\
& \bar{N}_{E}\left(r, \frac{1}{F-1}\right)=\bar{N}_{E}\left(r, \frac{1}{G-1}\right), \\
& \bar{N}_{L}\left(r, \frac{1}{F-1}\right)=\bar{N}_{S}\left(r, \frac{1}{G-1}\right) .
\end{aligned}
$$

Lemma 3. Suppose that $F$ and $G$ are two nonconstant meromorphic functions such that $F$ and $G$ share 1 IM, then

$$
\begin{aligned}
& \bar{N}_{L}\left(r, \frac{1}{F-1}\right) \leq \bar{N}\left(r, \frac{1}{F}\right)+\bar{N}(r, F)+S(r, F), \\
& \bar{N}_{L}\left(r, \frac{1}{G-1}\right) \leq \bar{N}\left(r, \frac{1}{G}\right)+\bar{N}(r, G)+S(r, G) .
\end{aligned}
$$

Proof. Obviously,

$$
\bar{N}_{L}\left(r, \frac{1}{F-1}\right) \leq N\left(r, \frac{1}{F-1}\right)-\bar{N}\left(r, \frac{1}{F-1}\right) \leq N_{0}\left(r, \frac{1}{F^{\prime}}\right) .
$$

From this and Lemma 2, we get (2.6). In the same way we can obtain (2.7).

Let

$$
F=f^{n} \quad \text { and } \quad G=g^{n},
$$

where $f$ and $g$ are nonconstant meromorphic functions, $n \geq 2$.

LEMMA 4. Let

$$
U=\frac{F^{\prime}}{F-1}-\frac{G^{\prime}}{G-1}
$$

where $F$ and $G$ are given by (2.8). If $U \equiv 0$, and $F$ and $G$ share $0 I M$, then $F \equiv G$.

Proof. Since $U \equiv 0$, by integration we have from (2.9) 


$$
F-1 \equiv A(G-1),
$$

where $A$ is a nonzero constant. We discuss the following two cases.

Case 1. Assume that 0 is not a lacunary value of $F$. Then there exists $z_{0}$ such that $F\left(z_{0}\right)=0$. Since $F$ and $G$ share $0 \mathrm{IM}$, we have $G\left(z_{0}\right)=0$. From $(2.10)$ we get $A=1$. Thus $F \equiv G$.

Case 2. Assume that 0 is a lacunary value of $F$. Since $F$ and $G$ share $0 \mathrm{IM}$, 0 is a lacunary value of $G$. If $A \neq 1$, from (2.10) we know that $1-A$ is a lacunary value of $F$. Noting $F=f^{n}$ and $n \geq 2$, we have

$$
\Theta(\infty, F) \geq \frac{n-1}{n} \geq \frac{1}{2}
$$

which is impossible. Thus, $1-A=0$. From this we obtain $F \equiv G$.

LEMMA 5. Suppose that $U$ is given by (2.9) and $U \neq \equiv$. If $F$ and $G$ share 0 $I M$, then

$$
(n-1) \bar{N}\left(r, \frac{1}{F}\right) \leq N(r, U)+S(r, F)+S(r, G)
$$

Proof. Since $F$ and $G$ share 0 IM, we know that $f$ and $g$ share 0 IM. Let $z_{0}$ be a zero of $f$ of order $p$, a zero of $g$ of order $q$. Then $z_{0}$ is a zero of $F$ of order $n p$, a zero of $G$ of order $n q$. From (2.9) we know that $z_{0}$ is a zero of $U$ of order at least $n-1$. From this we have

$$
(n-1) \bar{N}\left(r, \frac{1}{F}\right) \leq N\left(r, \frac{1}{U}\right) \leq T(r, U)+O(1) .
$$

By (2.9) we have

$$
m(r, U)=S(r, F)+S(r, G) .
$$

Combining this and (2.12) we obtain (2.11).

LEMMA 6. Assume that conditions of Lemma 5 are satisfied.

(1) If $F$ and $G$ share 1 and $\infty C M$, then

$$
(n-1) \bar{N}\left(r, \frac{1}{F}\right)=S(r) \text {. }
$$

(2) If $F$ and $G$ share $1 C M$ and $\infty I M$, then

$$
(n-1) \bar{N}\left(r, \frac{1}{F}\right) \leq \bar{N}(r, F)+S(r) .
$$

(3) If $F$ and $G$ share $1 I M$ and $\infty C M$, then

$$
(n-3) \bar{N}\left(r, \frac{1}{F}\right) \leq 2 \bar{N}(r, F)+S(r) .
$$


(4) If $F$ and $G$ share 1 and $\infty I M$, then

$$
(n-3) \bar{N}\left(r, \frac{1}{F}\right) \leq 3 \bar{N}(r, F)+S(r) \text {. }
$$

Proof. (1) From (2.9) we have

$$
N(r, U)=0 .
$$

From this and (2.11) we get (2.13).

(2) From (2.9) we have

$$
N(r, U) \leq \bar{N}(r, F) .
$$

From this and (2.11) we get (2.14).

(3) From (2.9) we have

$$
N(r, U)=\bar{N}_{L}\left(r, \frac{1}{F-1}\right)+\bar{N}_{L}\left(r, \frac{1}{G-1}\right) .
$$

By Lemma 3 we can obtain (2.6) and (2.7). From (2.6), (2.7) and (2.17) we get

$$
N(r, U) \leq 2 \bar{N}\left(r, \frac{1}{F}\right)+2 \bar{N}(r, F)+S(r)
$$

From this and (2.11) we get (2.15).

(4) From (2.9) we have

$$
N(r, U) \leq \bar{N}(r, F)+\bar{N}_{L}\left(r, \frac{1}{F-1}\right)+\bar{N}_{L}\left(r, \frac{1}{G-1}\right) .
$$

From (2.6), (2.7) and (2.18) we get

$$
N(r, U) \leq 2 \bar{N}\left(r, \frac{1}{F}\right)+3 \bar{N}(r, F)+S(r)
$$

From this and (2.11) we get (2.16).

LEMMA 7. Let

$$
V=\left(\frac{F^{\prime}}{F-1}-\frac{F^{\prime}}{F}\right)-\left(\frac{G^{\prime}}{G-1}-\frac{G^{\prime}}{G}\right)
$$

where $F$ and $G$ are given by (2.8). If $V \equiv 0$, and $F$ and $G$ share $\infty I M$, then $F \equiv G$.

Proof. Since $\mathrm{V} \equiv 0$, by integration we have from (2.19)

$$
1-\frac{1}{F} \equiv B-\frac{B}{G}
$$

where $B$ is a nonzero constant. We discuss the following two cases. 
Case 1. Assume that $\infty$ is not a lacunary value of $F$. Then there exists $z_{0}$ such that $1 / F\left(z_{0}\right)=0$. Since $F$ and $G$ share $\infty \mathrm{IM}$, we have $1 / G\left(z_{0}\right)=0$. From (2.20) we get $B=1$. Thus $F \equiv G$.

Case 2. Assume that $\infty$ is a lacunary value of $F$. Since $F$ and $G$ share $\infty$ IM, $\infty$ is a lacunary value of $G$. If $B \neq 1$, from $(2.20)$ we know that $1 /(1-B)$ is a lacunary value of $F$. Noting $F=f^{n}$ and $n \geq 2$, we have

$$
\Theta(0, F) \geq \frac{n-1}{n} \geq \frac{1}{2},
$$

which is impossible. Thus, $B=1$. From this we obtain $F \equiv G$.

Lemma 8. Suppose that $V$ is given by (2.19) and $V \not \equiv 0$. If $F$ and $G$ share $\infty$ $I M$, then

$$
(n-1) \bar{N}(r, F) \leq N(r, V)+S(r, F)+S(r, G) .
$$

Proof. Since $F$ and $G$ share $\infty$ IM, we know that $f$ and $g$ share $\infty$ IM. Let $z_{0}$ be a pole of $f$ of order $p$, a pole of $g$ of order $q$. Then $z_{0}$ is a pole of $F$ of order $n p$, a pole of $G$ of order $n q$. From (2.19) we have

$$
V=\frac{F^{\prime}}{F(F-1)}-\frac{G^{\prime}}{G(G-1)}
$$

From this we know that $z_{0}$ is a zero of $V$ of order at least $n-1$. Thus,

$$
(n-1) \bar{N}(r, F) \leq N\left(r, \frac{1}{V}\right) \leq T(r, V)+O(1) .
$$

By (2.19) we have

$$
m(r, V)=S(r, F)+S(r, G) .
$$

Combining this and (2.22) we obtain (2.21).

Using Lemma 8 and proceeding as in the proof of Lemma 6, we can prove the following lemma.

Lemma 9. Assume that conditions of Lemma 8 are satisfied.

(1) If $F$ and $G$ share 1 and $0 C M$, then

$$
(n-1) \bar{N}(r, F)=S(r) .
$$

(2) If $F$ and $G$ share $1 C M$ and $0 I M$, then

$$
(n-1) \bar{N}(r, F) \leq \bar{N}\left(r, \frac{1}{F}\right)+S(r)
$$

(3) If $F$ and $G$ share $1 I M$ and $0 C M$, then

$$
(n-3) \bar{N}(r, F) \leq 2 \bar{N}\left(r, \frac{1}{F}\right)+S(r) .
$$


(4) If $F$ and $G$ share 1 and $0 I M$, then

$$
(n-3) \bar{N}(r, F) \leq 3 \bar{N}\left(r, \frac{1}{F}\right)+S(r) .
$$

LEMMA 10 (see [13] or [14]). Let $F$ and $G$ be two nonconstant meromorphic functions such that $F$ and $G$ share 1 IM. If

$$
\bar{N}\left(r, \frac{1}{F}\right)+\bar{N}(r, F)=S(r, F) \text { and } \bar{N}\left(r, \frac{1}{G}\right)+\bar{N}(r, G)=S(r, G),
$$

then $F \equiv \mathrm{G}$ or $F \cdot G \equiv 1$.

Remark. From Lemma 10 we immediately deduce the following result: If

Suppose that $F$ and $G$ are given by (2.8), and $F$ and $G$ share 1, 0 and $\infty$ IM.

$$
\bar{N}\left(r, \frac{1}{F}\right)+\bar{N}(r, F)=S(r),
$$

then $f \equiv t g$, where $t^{n}=1$ or $f \cdot g \equiv s$, where 0 and $\infty$ are lacunary values of $f$ and $g$, and $s^{n}=1$.

This observation will be used in several proofs of our Theorems.

\section{Proof of main results}

3.1. Proof of Theorem 1. Let $F$ and $G$ be given by (2.8). If $F \equiv G$, then $f \equiv t g$, where $t^{n}=1$. Thus, Theorem 1 holds. Next, we suppose $F \neq G$. Since $f$ and $g$ share the sets $S_{1}$ and $S_{3} \mathrm{CM}$ and $S_{2} \mathrm{IM}$, we know from (2.8) that $F$ and $G$ share the values 1 and $\infty \mathrm{CM}$ and $0 \mathrm{IM}$. Let $U$ and $V$ be given by (2.9) and (2.19) respectively. Noting $F \neq G$, by Lemma 4 and Lemma 7 we have $U \neq 0$ and $V \not \equiv 0$. By Lemma 6 and Lemma 9 we can obtain (2.13) and (2.24). Noting $n \geq$ 2 , from (2.13) and (2.24) we can obtain (2.27). By Lemma 10 we obtain the conclusion of Theorem 1 .

3.2. Proof of Theorem 2. Let $F$ and $G$ be given by (2.8). If $F \equiv G$, then $f \equiv t g$, where $t^{n}=1$. Thus, Theorem 2 holds. Next, we suppose $F \equiv G$. Since $f$ and $g$ share the sets $S_{2}$ and $S_{3}$ IM and $S_{1} \mathrm{CM}$, we know from (2.8) that $F$ and $G$ share the values 0 and $\infty \mathrm{IM}$ and $1 \mathrm{CM}$. Let $U$ and $V$ be given by (2.9) and (2.19) respectively. By Lemma 6 and Lemma 9 we can obtain (2.14) and (2.24). Noting $n \geq 3$, from (2.14) and (2.24) we can obtain (2.27). By Lemma 10 we obtain the conclusion of Theorem 2 .

3.3. Proof of Theorem 3. Let $F$ and $G$ be given by (2.8). If $F \equiv G$, then $f \equiv t g$, where $t^{n}=1$. Thus, Theorem 3 holds. Next, we suppose $F \neq G$. Since $f$ and $g$ share the sets $S_{1}$ and $S_{3} \mathrm{IM}$ and $S_{2} \mathrm{CM}$, we know from (2.8) that $F$ and $G$ 
share the values 1 and $\infty \mathrm{IM}$ and $0 \mathrm{CM}$. Let $U$ and $V$ be given by (2.9) and (2.19) respectively. By Lemma 6 and Lemma 9 we can obtain (2.16) and (2.25). Noting $n \geq 6$, from (2.16) and (2.25) we can obtain (2.27). By Lemma 10 we obtain the conclusion of Theorem 3 .

3.4. Proof of Theorem 4. Let $F$ and $G$ be given by (2.8). If $F \equiv G$, then $f \equiv t g$, where $t^{n}=1$. Thus, Theorem 4 holds: Next, we suppose $F \equiv \equiv$. Since $f$ and $g$ share the sets $S_{1}$ and $S_{2} \mathrm{IM}$ and $S_{3} \mathrm{CM}$, we know from (2.8) that $F$ and $G$ share the values 1 and $0 \mathrm{IM}$ and $\infty \mathrm{CM}$. Let $U$ and $V$ be given by (2.9) and (2.19) respectively. By Lemma 6 and Lemma 9 we can obtain (2.15) and (2.26). Noting $n \geq 6$, from (2.15) and (2.26) we can obtain (2.27). By Lemma 10 we obtain the conclusion of Theorem 4 .

3.5. Proof of Theorem 5. Let $F$ and $G$ be given by (2.8). If $F \equiv G$, then $f \equiv t g$, where $t^{n}=1$. Thus, Theorem 5 holds. Next, we suppose $F \neq \equiv$. Since $f$ and $g$ share the sets $S_{1}, S_{2}$ and $S_{3}$ IM, we know from (2.8) that $F$ and $G$ share the values 1,0 and $\infty$ IM. Let $U$ and $V$ be given by (2.9) and (2.19) respectively. By Lemma 6 and Lemma 9 we can obtain (2.16) and (2.26). Noting $n \geq 7$, from (2.16) and (2.26) we can obtain (2.27). By Lemma 10 we obtain the conclusion of Theorem 5 .

3.6. Proof of Theorem 6. Let $F$ and $G$ be given by (2.8). If $F \equiv G$, then $f \equiv t g$, where $t^{n}=1$. Thus, Theorem 6 holds. Next, we suppose $F \neq G$. Since $f$ and $g$ are nonconstant entire functions such that $f$ and $g$ share the sets $S_{1}, S_{2}$ IM, we know from (2.8) that $F$ and $G$ are nonconstant entire functions such that $F$ and $G$ share the values 1 and 0 IM. Let $U$ and $V$ be given by (2.9) and (2.19) respectively. By Lemma 6 we can obtain

$$
(n-3) \bar{N}\left(r, \frac{1}{F}\right)=S(r) \text {. }
$$

Noting $n \geq 4$, from (3.1) we can obtain (2.27). By Lemma 10 we obtain the conclusion of Theorem 6 .

\section{Concluding Remarks}

4.1. Remark 1. In the same manner as the above, it is easy to give the proofs of Theorem $\mathrm{C}$ and Theorem $\mathrm{D}$. Next we proceed to prove Theorem $\mathrm{D}$.

Let $F$ and $G$ be given by (2.8). If $F \equiv G$, then $f \equiv t g$, where $t^{n}=1$. Thus, Theorem D holds. Next, we suppose $F \neq \equiv$. Since $f$ and $g$ share the sets $S_{1}$ and $S_{2} \mathrm{CM}$ and $S_{3}$ IM, we know from (2.8) that $F$ and $G$ share the values 1 and 0 $\mathrm{CM}$ and $\infty$ IM. Let $U$ and $V$ be given by (2.9) and (2.19) respectively. By Lemma 6 and Lemma 9 we can obtain (2.14) and (2.23). Noting $n \geq 2$, from (2.14) and (2.23) we can obtain (2.27). By Lemma 10 we obtain the conclusion 


\section{of Theorem D.}

4.2. Remark 2. It is clear that Theorem 1 follows from Theorem $D$ by letting $f \rightarrow 1 / f$ and $g \rightarrow 1 / g$, that Theorem $\mathrm{D}$. follows from Theorem 1 by letting $f \rightarrow 1 / f$ and $g \rightarrow 1 / g$. Thus, Theorem $\mathrm{D}$ and Theorem 1 are equivalent to each other. Similarly, Theorem 3 and Theorem 4 are equivalent to each other, and also Lemma 6 and Lemma 9.

4.3. Remark 3. Let $f(z)=1-3 e^{z}+3 e^{2 z}-e^{3 z}$ and $g(z)=3 e^{-z}-3 e^{-2 z}$. It is easy to see that this example shows that the assumption " $n \geq 2$ " in Theorem 1 is best possible. However, whether the assumption " $n \geq 3$ " in Theorem 2 , the assumption " $n \geq 6$ " in Theorem 3 , Theorem 4 and its corollary, the assumption " $n \geq 7$ " in Theorem 5 , the assumption " $n \geq 4$ " in Theorem 6 are best possible or not, is still an open question to be resolved.

Acknowledgement. I am grateful to the referee for valuable suggestions and comments.

\section{REFERENCES}

[1] W. K. Hayman, Meromorphic Functions, Clarendon Press, Oxford, 1964.

[2] F. Gross, On the distribution of values of meromorphic functions, Trans. Amer. Math. Soc., 131 (1968), 199-214.

[3] G. G. Gundensen, Meromorphic functions that share three or four values, J. London Math. Soc., 20 (1979), 457-466.

[4] F. Gross And C. F. Osgood, Entire functions with common preımages, Factorization Theory of Meromorphic Functions, Marcel Dekker, 1982, 19-24.

[5] H.-X. YI, Meromorphic functions with common preımages, J. of Math. (Wuhan), 7 (1987), 219-224.

[6] G. Brosch, Eindeutigkeıtssätze für meromorphe Funktionen, Thesis, Technıcal Universıty of Aachen, 1989.

[7] H.-X. Yl, On the unıqueness of meromorphıc functions, Acta Math. Sinica, 31 (1988), $570-576$.

[ 8 ] K. Toнge, Meromorphic functions covering certain finite sets at the same points, Kodai Math. J., 11 (1988), 249-279.

[ 9 ] H.-X. Yı, Meromorphic functions that share two or three values, Kodaı Math. J., 13 (1990), $363-372$.

[10] G. Jank ANd N. Terglane, Meromorphic functions sharing three values, Math. Pannon., 2 (1991), 37-46.

[11] L. R. Sons, Deficiencies of monomials, Math. Z., 111 (1969), 53-68.

[12] S.-P. WANG, On meromorphic functions that share four values, J. Math. Anal. Appl., 173 (1993), 359-369.

[13] H.-X. YI AND C. C. YANG, Theory of the Unıqueness of Meromorphic Functions, Science Press, Beijing, 1995.

[14] R. Nevanlinna, Einige Eindeutigkeitssätze in der Theorie der meromorphen Funktionen, Acta Math., 48 (1926), 367-391. 
Department of Mathematics

SHANDONG UNIVERSITY

JiNAN, ShaNDONG 250100

People's Republic of China 